\title{
The Brain-Gut Axis: From Pathophysiology to Possible Future Strategies of Treatment
}

\section{Simone Vigneri*}

Department of Experimental Biomedicine and Clinical Neuroscience (BioNeC), University of Palermo, Italy

${ }^{*}$ Corresponding author: Simone Vigneri, MD, Department of Experimental Biomedicine and Clinical Neuroscience (BioNeC), University of Palermo, Via Gaetano La Loggia 1, 90129, Palermo, Italy, Tel: +39 091 6555105; Fax: +39 091 6555102; E-mail: simone.vigneri@gmail.com

Received date: Jun 17, 2014, Accepted date: Aug 4, 2014, Published date: Aug 14, 2014

Copyright: (C) 2014 Vigneri S. This is an open-access article distributed under the terms of the Creative Commons Attribution License, which permits unrestricted use, distribution, and reproduction in any medium, provided the original author and source are credited.

\begin{abstract}
The enteric nervous system (ENS) controls and modulates various functions of the digestive tract such as motility, exocrine and endocrine secretion, local microcirculation, inflammation and neuroimmunity. This system is autonomous from the central nervous system (CNS), although they share many neurons and neurotransmitters. The CNS may exert a modulatory role on ENS through brainstem and subcortical areas, peripheral and autonomic fibers. The brain-gut axis is the complex system regulating the interactions and mutual influences between the nervous system and the gut. This axis is divided into various levels through receptors, afferents, integration centers, efferents and effectors, and exploits multiple messengers belonging to the nervous, immune and endocrine systems. The proper functioning of this apparatus is essential in intestinal physiology, its involvement has been recognized as one of the leading pathophysiological basis of many digestive disorders and a target for current and future therapeutical approaches.
\end{abstract}

Keywords: Brain gut; Enteric nervous system; Dysmotility; Central modulation; Neuroimmunity; Microbiota; Hyperalgesia; Appetite

\section{Introduction}

The gastrointestinal tract is an extended system with a complex and critical multi-level control. Despite the growing importance and autonomy attributed to the enteric nervous system (ENS), an important role is played by other areas of the nervous system whose dysfunction at any level may result in disorders of the digestive tract. This review describes the essential components and pathophysiology of the brain-gut axis involved in the origin of several gastrointestinal system disorders, the available treatments and future therapeutical perspectives.

\section{The brain-gut axis}

The brain-gut axis (BGA) is defined as a complex system which regulates interactions and mutual influences between the nervous system and the gut. This axis is divided into various levels through receptors, afferents, integration centers, efferents and effectors, and exploits multiple messengers belonging to the nervous, immune and endocrine system. It owns a hierarchical organization whose apex is the central autonomic network, leading integration of afferents and autonomic responses to different stimuli [1]. The prefrontal cortex (PFC) plays an important modulatory effect on the anterior cingulate (ACC) and insular cortex (IC). The IC represents the sensory visceral cortex, receives visceral nociceptive inputs and helps modulating autonomic functions such as heart rate, blood pressure, respiration and gastrointestinal motility [2]. ACC responds to nociceptive stimuli (motor visceral area), regulates neuroendocrine function (through interconnection with the hypothalamus) and modulates emotional responses (synergistic action with the amygdala) [3]. Ponto-bulbar nuclei tonically inhibit these reflexes while prefrontal areas regulate the sympatho-vagal and the medullary output through descending pathways, modulating the response to environmental stimuli. The periaqueductal gray (PAG) is an important structure for coordination and control of visceral pain. The nucleus of the solitary tract (NTS) is the main brainstem site for the arrival of afferents from the periphery. Through vagal fibers, stimuli from the intestinal environment reach NTS which, in turn, project to the dorsal motor nucleus of the vagus (DMN), dealing with intestinal motor reflexes. Although mechanical or chemical stimuli can activate enteric local circuits, most of responses involve mesenteric (via mesenteric ganglia) or vago-vagal (via the DMN) reflexes [4].

The intestinal innervation is ensured by extrinsic and intrinsic innervation, whereas the extrinsic component acts by modulating the latter:

Extrinsic innervation: Mainly the vagus nerve, whose afferent branch essentially consists of myelinated fibers with their soma located in the nodose ganglion and terminating in the NTS. The vagal afferent fibers are sensitive to mechanical stimuli and mediate the sense of satiety and nausea. Moreover, it seems involved in the modulation of pain due to excitatory or inhibitory action on spinal nociceptive pathways. The efferent motor component includes the nucleus ambiguous (NA) and the DMN. NA is involved in the motor activity of the proximal portion of the digestive tract while $\mathrm{DMN}$ regulates intestinal activity due to synaptic junction with the myenteric plexus. In addition, the DMN carries fibers to the NTS allowing quick vagovagal reflex arcs. In the brainstem, NTS and DMN form a functional unit with overlapping areas but high heterogeneity and selectivity of reflex responses (e.g., entero-gastric or gastro-colic reflex) [5].

The CNS is an additional control site of bowel function through its modulatory action on the brainstem. Long fibers can lead the transmission from NTS to thalamus and hypothalamus, limbic cortex and insula. The motor nuclei of the vagus are related with brain areas such as the area postrema, hypothalamus, orbitofrontal and anterior cingulate cortices [6]. This hierarchical and branched organization 
Page 2 of 7

allows not only the start of visceral reflexes, but also the simultaneous participation of the cardiovascular and respiratory system in the course of autonomic responses.

The spinal innervation is provided by visceral afferents that reach the dorsal horns of the spinal cord through pre and paravertebral ganglia, co-localizing with somatic fibers (this mechanism is the basis of referred pain). Visceral fibers mainly follow the lateral (discrimination of pain) and medial (emotional component of pain) spinothalamic path, although the involvement of dorsal columns or accessory pathways such as the spinoreticular has been postulated [7].

The efferents rise from preganglionic cholinergic fibers in the intermediolateral thoraco-lumbar columns and synapse in ganglia (i.e., the superior mesenteric/celiac and inferior ganglia for small and large intestine, the pelvic ganglion for rectum). Noradrenergic postganglionic fibers innervate ganglia of the myenteric plexus by inhibiting the release of acetylcholine from myenteric and submucosal neurons, thereby reducing peristalsis. Either inhibitory reflexes and CNS modulation occur at this level as well (e.g., the hypothalamic stimulation increases whereas the orbital and cingulate cortex activation inhibits colonic motility) [6].

Finally, pelvic ganglia and sacral nerves innervate the distal colon, rectum, and internal anal sphincter. Afferents to the sacral cord come from pelvic and pudendal nerves through the dorsal root ganglia and allow the trigger of reflexes such as defecation [8].

Intrinsic innervation: based on the myenteric and submucosal plexus of the ENS, consisting of approximately 100 million neurons. Although under control of the CNS and SNA, it is an autonomous system mostly dependent on intrinsic primary afferent neurons (IPAN ) and interneurons.

The Auerbach's myenteric plexus lies between the layers of longitudinal and circular muscle, receives vagal modulating efferents and is responsible for muscle tone and peristaltic activity of the gut. The Meissner's submucosal plexus lies in the submucosal layer and is more developed in the small intestine. It seems involved in the control of ion transport and secretion, and in the maintenance of blood flow, receives sensory afferents from chemoreceptors and mechanoreceptors located along the whole digestive tract. The myenteric and submucosal IPAN within the ENS transmit informations about the mechanical distortion of external muscle layers and mucosa respectively, and the changes in $\mathrm{pH}$ within the intestinal lumen. Extrinsic primary afferent neurons (EPAN) are responsible for the transmission of afferents to the brain and the subsequent perception of nausea or satiety.

ENS plays a central role in many functions throughout the gastrointestinal tract: tonic inhibition of the contraction, mix of the intestinal content, propagation of the motor migrating complex, reflex peristaltic activity, modulation of the sphincter tone, secretion into the intestinal lumen, changes in the microcirculation, activation of flogosis [9].

\section{The Brain-Gut Pathophysiology}

Several factors are involved in the brain-gut pathophysiology:

- Dysmotility

- Stress

- Visceral hypersensitivity

- Impaired central modulation

- Appetite dysregulation

\section{- Neuroimmunity \\ - Neuropeptides \\ - Altered gut microbiota}

Dysmotility: Ineffective peristalsis can be primary or secondary to systemic diseases. A disabling disease with poor prognosis and often misunderstood is chronic intestinal pseudo-obstruction. Mostly idiopathic, it recognizes three basic variants: neuropathic, myopathic and mesenchymal. The first requires an involvement of the intestinal plexi (anti-neuronal and anti-Hu positive antibodies), the second form is associated with the involvement of muscle layers (altered expression of smooth muscle-specific actin and desmin) and normal enteric innervation, the latest form is due to alteration of the interstitial cells of Cajal [10].

Hirschsprung's disease is a congenital aganglionosis of the myenteric and submucosal plexus in the distal colon and rectum that causes impaired transit and risk of megacolon. The main responsible mutation is in the RET gene, encoding a receptor for the glial cellderived neurotrophic factor, and whose alterations are associated with abnormal embryological development of the neural crest cells [11]. Moreover, intestinal hypoganglionosis and neuronal dysplasia (hyperganglionosis with non-functional cells) are included in the dysganglionosis too.

In irritable bowel syndrome (IBS) up to $75 \%$ of patients show intestinal movement disorders manifest either as a reduction or increase of peristalsis. The diarrhea pattern includes an increase of peristaltic waves and reduced transit time; the constipation pattern shows reduction of peristaltic waves, increased transit time and hard consistency of stool; abdominal pain is a symptom common to the two patterns. Dysmotility may be related to the reduced nociceptive threshold and increased perception of pain in these patients: slow intestinal transit promotes the accumulation of gas and the perception of abdominal distension as reduced sensory threshold makes patients susceptible to motor migrating complexes [12].

Stress: Stress represents the body's response to stimuli. Although physiological, it can become pathological in case of low adaptive capacity of the organism to environmental stimuli. The stress-induced changes are implemented via the hypothalamic-pituitary-adrenal axis (HPA) stimulated by the release of molecules such as corticotropinreleasing factor (CRF). The HPA reacts to stimuli which alter the intestinal environment and reach the CNS, triggering responses in order to bring the system back to its equilibrium. Any damage able to activate the intestinal sensory afferents can promote the release of CRF, promoting degranulation of mast cells and macrophages, monocyte chemotaxis and release of proinflammatory cytokines [13].

The activation of the immune system, in turn, causes an increase in sympathetic tone with release of catecholamines and inhibition of vagal activity (which modulates the immune system), configuring an "inflammatory status" [14]. The balance between sympathetic and vagal tone is modulated by cortico-subcortical areas such as PFC and amygdala. The latter is stimulated under stress and modulated by input from the PFC which trigger a vagal inhibitory feedback on the HPA, reducing the release of cortisol and the heart rate variability [15]. On the other hand, chronic stress may be responsible for maladaptive changes: in experimental conditions, the development of chronic colitis may cause the reduced expression of the CRF gene and cortisol blood levels associated with blunt activation of the HPA and greater susceptibility to infections [16]. CRF receptors are also distributed along the digestive tract on the surface of immune cells and their 
ligands may modulate proinflammatory (CRF1) or anti-inflammatory (CRF2) shifts [17].

Last, psychological trauma in early life or psychiatric syndromes in adulthood can affect the neuroendocrine system. In animal models, the early maternal separation was found to be associated with hyperactivity of the HPA, visceral hyperalgesia, diarrhea, intestinal barrier dysfunction and anxiety [18]. The association between depression and immune activation in some cases has been questioned. Nevertheless, the association between depressive-like behaviour and development of colitis responsive to tricyclic drugs has been confirmed [19].

Visceral hypersensitivity: The detection of visceral hypersensitivity and reduced threshold in response to painful stimuli (e.g., rectal distension) is common in patients with IBS. Its frequency in various studies varies between $57 \%$ and $90 \%$ of patients: the association is confirmed by studies showing the rise of gastrointestinal symptoms during the menstrual phase, when the concentration of circulating sex hormones with analgesic effects tend to decrease [20]. Sensitization may arise from ENS to cortex: the prolonged exposure of the mucosa to persistent inflammatory noxae can determine increased permeability of the intestinal barrier, synthesis of cytokines and molecules able to maintain inflammation (e.g., nitric oxide inducible synthase), changes in the transmission and integration of afferent stimuli to the cortex, hypervigilance, neuroendocrine, autonomic and behavioral dysfunctions. These changes are the result of synaptic plasticity involving altered transcription and protein synthesis, and can justify the persistent organism activation even in the absence of noxious stimuli [21]. Therefore, chronicization implies modifications beyond mere temporal criteria and may explain the frequent failure of conventional therapies.

Impaired central modulation: reciprocal interactions of hypothalamus, amygdala and locus coeruleus (LC) provide evidence of a positive feedback between the HPA and the ascending noradrenergic circuits responsible for arousal. By virtue of the LC connections with visceral sensory and motor nuclei, chronic stress may persistently activate the HPA and affect intestinal activity. Brain areas involved in the regulation of mood (and visceral sensitivity) as hippocampus and PFC have a high density of cortisol receptors: variations in circulating levels of cortisol observed in psychiatric disorders such as major depression are associated with plastic changes in these structures [22].

Pain is the main symptom of functional gastrointestinal disorders (FGID). The development of functional imaging such as positron emission tomography and functional magnetic resonance imaging allowed the identification of brain areas involved in perception and reaction to visceral noxious stimuli. These areas are part of the "pain matrix": the primary and secondary somatosensory cortex and IC, involved in discrimination of visceral pain stimulus, the PFC and posterior parietal cortex mediate the cognitive component of pain, the ACC responsible for the affective-emotional dimension in response to nociception. The ACC is also described as the main descending pain modulatory area ("pain inhibitory system"), a control system often impaired in gastrointestinal diseases [23]. Amygdala and PAG receive afferents from the ACC and send axons to brainstem areas able to inhibit the nociceptive transmission to the spinal cord or they may promote the release of endogenous opioids and analgesic neurotransmitters (e.g., 5-HT, NA) [24].

The primary cause of peripheral sensitization is an inflammatory trigger of the immune system: The release of proinflammatory cytokines, interleukins and prostaglandins sensitizes peripheral receptors promoting their activation for sub-threshold stimuli as well as the opening of ion channels previously inactive and the increased expression of genes and protein synthesis. Central sensitization is based on increased membrane excitability and discharge of dorsal horn neurons, expression of silent genes with synthesis of neurotransmitters and receptors, activation of NMDA receptors, morphological changes (e.g., widened cortical excitability area). These processes have important clinical implications being the basis of phenomena such as allodynia and the ineffectiveness of conventional treatment in chronic pain.

Electroencephalography has been recently applied in research. The high temporal resolution of this technique allows to associate it with other tools such as neurophysiological evoked potentials to assess variation and cortical topography of the frequency bands after gut painful stimuli [25]. Rossel et al. have documented a reduction in latency and amplitude of evoked potentials in IBS patients compared to controls: these results confirm the role of neuronal plasticity in the pathophysiology of this disease [26].

Appetite dysregulation: Appetite is an essential function, responsible of the balance between energy intake and expenditure in the human body. Its regulation is maintained by the interaction of CNS and ENS, ensured by communications of sensory afferents spreading from the gut and parasympathetic/sympathetic efferents. Different stimuli such as mechanical, chemical or neurohormonal can trigger short- or long-distance responses. Local responses are conveyed within the ENS through intramural motor, secretory or vascular reflexes whereas CNS-mediated responses are activated through spinal or brainstem reflexes (whose afferents are spinal or vagal nerves), as mentioned in previous paragraphs. The increased expression of genes like c-fos seems related to vagal signaling from gut to brain and gastrointestinal activation (e.g., after gastric distension or cholecystokinin secretion). This complex system regulates the food intake and control of body weight either with short-term (meal-tomeal) and long-term (neurohormonal control).

In the peripheral signaling pathway, several neuropeptides affect the food intake by increasing (e.g., ghrelin, orexins/OX) or decreasing (cholecystokinin/CCK, leptin, oxyntomodulin/OXM) appetite. These molecules act stimulating or inhibiting the expression and release in the hypothalamic arcuate nucleus of neuropeptide $\mathrm{Y}$ and agoutirelated protein, which in turn, activate the paraventricular nuclei promoting the release of thyrotropin releasing hormone (TRH), corticotropin releasing hormone (CRH) and oxytocine. Short-term regulation lies on gut hormones such as CCK and OXM, whereas the long-term pathway mostly depends upon the release of leptin by the fat tissue, promoting satiety and whose action is balanced by ghrelin activity ("ghrelin-leptin tango") [27].

Any impairment or dysregulation of neuropeptide secretion as well as of hypothalamic, brainstem or spinal areas involved in these processes might alter food intake causing imbalance between energy intake and expenditure. For instance, in obese subjects alterations in ghrelin, leptin, insulin levels as well as hyperactivation of orexinergic endocannobinoid system or mutations of anorexinergic receptors such as MC3R and MC4R have been reported in many studies [28].

Neuroimmunity: The role of the immune system has already been reported as a main actor of BGA pathophysiology and sensitization: for example, the high frequency of post-infectious IBS or the reduced expression of anti-inflammatory cytokines (e.g., IL-10, TGF) in these 
patients. Although normal endoscopic examination, frequent evidence of low-grade mucosal inflammation (increased mast cells in the muscularis mucosae of ileum and colon) and infiltration of Tlymphocytes in the myenteric plexus has been documented in IBS, in contrast with previous theories considering this disorder sine materia. Predisposing factors (e.g., genetic, previous gastroenteritis, food allergies, intestinal dysmicrobism) may favor the onset of inflammatory status and the sensitization of IPAN and EPAN, resulting in visceral hypersensitivity and motility disorders [29].

Neuropeptides: Although in the past it was believed that acetylcholine (Ach) and serotonin (5-HT) constituted the whole pool of ENS neurotransmitter, recently an increasing number of molecules was added: GABA, norepinephrine (NE), vasoactive intestinal peptide (VIP), nitric oxide (NO), CCK, opioids, substance P, somatostatin, gene-related peptide to calcitonin (CGRP) and ATP among the most studied.

The $95 \%$ of $5-\mathrm{HT}$ is stored in the intestinal enterochromaffin cells (EC), which are important for the initiation of peristaltic and secretory reflexes. Enteroendocrine cells and EC are stimulated by $\mathrm{pH}$ changes in the intestinal lumen and an altered release of neurotransmitters can result in the pathological activation of intrinsic and extrinsic afferent nerve endings. EC microvilli stimulate the release of $5-\mathrm{HT}$ in the lumen or in the lamina propria thanks to afferent fiber activation, and promote secretion and propulsive activity. The central role of 5-HT in the pathophysiology of ENS is confirmed by higher EC and 5-HT concentration in patients with IBS.9 This may depend on: 1) increased release of 5-HT from nerve terminals leading to pathological peristalsis and recruitment of lymphocytes through the endothelium; 2) reduced reuptake of 5-HT from the synaptic cleft (possible role of the serotonine transporter gene polymorphism) [30].

The increased knowledge on the neurochemistry of ENS have led to revise old assumptions mainly focusing on glutamatergic and GABAergic systems. An important role in the physiology of the vagovagal reflexes is played by non-adrenergic non-cholinergic (NANC) fibers: GABA neurons in the NTS may interact either with excitatory cholinergic and inhibitory NANC fibers in the DMN, suggesting complex homeostatic mechanisms [31]. The knowledge of intestinal neurochemistry has a great importance in the choice of a proper treatment: for example, the use of 5-HT3 receptor antagonists should be preferred in IBS with predominant diarrhea whereas 5-HT4 agonists when constipation is prevailing [32].

Altered gut microbiota: The digestive tract hosts high concentrations of different microorganisms (more than 50 types and about 1014 cells per gram of intestinal content), according to the intestinal site [33]. The gut microbiota composition is regulated by several factors: intrinsic factors such as genetic predisposition, $\mathrm{pH}$ (high concentrations of $\mathrm{H}+$ in the gastric lumen) and concentrations of $\mathrm{O}_{2}$ (the number of anaerobes increases in the colon), peristalsis, the degree of mucous secretion, the presence of $\operatorname{IgA}$; extrinsic factors such as diet, stress, the use of probiotics or drugs (e.g., antibiotics, proton pump inhibitors, prokinetics, opioids or nonsteroidal antiinflammatory drugs) or infections.

An abnormal proliferation of the flora is accompanied by the production and release of bacterial peptides, proinflammatory cytokines from lymphocytes and dendritic cells, and of 5-HT from the EC. In IBS, these molecules promote the outer membrane barrier permeabilization and the passage into the blood circulation of proinflammatory substances, triggering CNS-mediated autonomic and behavioral responses responsible for the long-lasting intestinal dysmicrobism and BGA imbalance [34]. The main bacteria found in the stool and mucosa of patients with IBS are coliforms, anaerobes, $E$. Coli, bacterioides, whereas mucosal lactobacilli and bifidobacteria seem to be reduced when diarrhea is predominant.

The altered microbiota is associated with increased production of intestinal gas, explaining one of the main IBS symptoms: bloating. Several treatments to restore the normal microbiota have been suggested: the use of gut-selective antibiotics for a short period in association with probiotics, the elimination of high fermentation and poor digestibility carbohydrates from the diet, the discontinuation of proton pump inhibitors.

\section{Therapeutical Strategies and Future Approaches}

Considering the complex pathophysiology, several approaches to evaluate and treat BGA dysfunctions have been recently reported in preclinical and clinical studies.

Non-invasive neurostimulation techniques have been used to investigate the pathophysiology of chronic pain and modulation of CNS. The transcranial magnetic stimulation (TMS) exploits the capacity of an electric current to generate an electromagnetic field delivered through a coil: the magnetic pulse creates a current flow in nearby tissues, managing to excite or inhibit specific cortical areas. Transcranial direct current stimulation (tDCS) applies constant currents of low intensity delivered through small electrodes on a brain site: compared to TMS, it owns a mere modulatory fuction, lower spatial resolution and exerts its effects either in the short term (within few minutes after stimulation) due to its effects on the membrane potential, and in the long term (within few hours after the end of the session) through plastic changes involving NMDA receptors, resembling the long-term potentiation/depression. The anodal stimulation increases while the cathodal reduces neuronal excitability of the stimulated areas [35]. Although some studies report an effect of TMS on perceived pain in patients with chronic pancreatitis, a Cochrane review suggested a potential but not yet significant role in the control of chronic pain after HF-rTMS and anodal tDCS on the motor cortex, requiring further investigations $[36,37]$.

In this sense, a recent work with tDCS applied on the motor cortex of patients affected by gastroesophageal reflux disease (GERD) found significant increase of distal wave amplitudes and improvement of pathological motor pattern only in subjects with non-erosive disease. Therefore, pathophysiological processes in GERD might be due not only to peripheral damage but to central neural control involvement as well. Erosive forms might hide more severe dysfunctions of the cortico-esophageal circuit in comparison with non-erosive subjects, highlighting the diffuse interaction between brain and ENS either in health and disease, and the chance to act on BGA through noninvasive transcranial stimulation (Figure 1), [38]. 


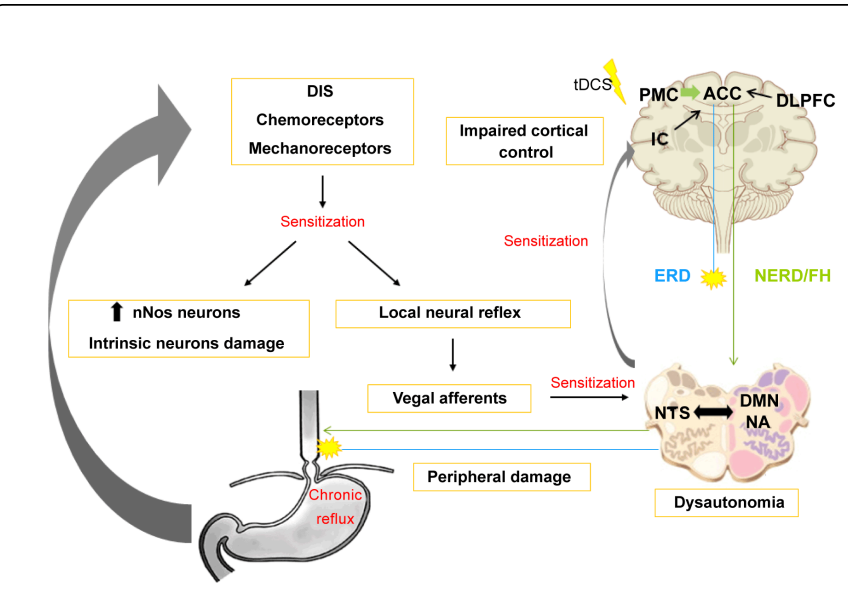

Figure 1: Hypothesis on the different effects of tDCS on nonerosive (NERD) vs. erosive (ERD) disease. DIS, dilated intercellular spaces; nNOS, neuronal nitric oxide synthase; tDCS, transcranial direct current stimulation; NTS, nucleus of tractus solitarius, DMN, dorsal motor nucleus of vagus; NA, nucleus ambiguous; PMC, premotor cortex; ACC, anterior cingulate cortex; DLPFC, dorsolateral prefrontal cortex; IC, insular cortex.

The fact that drugs acting on the CNS have been proven to be effective in FGID support the major role of brain and neurotransmitter dysfunctions in BGA pathophysiology. Although convincing clinical trials supporting the efficacy of these drugs are still lacking, the effectiveness of antidepressants (tricyclics and SSRI), anxyolitics and sedatives might be related to sleep restoration and pain improvement, anticholinergic effects, normalization of gastrointestinal transit, fundic relaxation and peripheral antineuropathic effects, not only to frequent psychiatric comorbidities in such patients. In agreement with recent studies, amytriptiline, desipramine, citalopram, fluoxetine and paroxetine are the mostly administered drugs in IBS, with a potentially effective role of serotonin and norepinephrine reuptake inhibitors (SNRIs) such as duloxetine [39].

Pediatric data suggest that hypnotherapy and cognitive behavioral therapy might be a useful intervention in children with recurrent abdominal pain and IBS, whereas chloride secretagogues can help constipation improvement and relief of abdominal pain [40]. Other medications such as 5-HT3 antagonists, rifaximin, probiotics have been used with variable efficacy and evidence. There are no current data on the effectiveness of the probiotic-prebiotics association [41]. The probiotics seem to have a mild positive effect in patients with IBS whereas promising therapeutic results were obtained after administration of Bifidobacterium bifidum MIMBb75 [42].

Among the new generation drugs, 5-HT3 antagonists (e.g., ramosetron) and 5HT4 agonists have promoted an accelerated gut transit in patients with chronic constipation in various studies but their safety profile still needs to be assessed. New intestinal secretagogues (e.g., linaclotide) and bile acid modulators (e.g.,colesevelam) appeared to be promising agents by accelerating or slowing colonic transit in IBS with constipation and diarrhea, respectively [40]. LX-1031, a tryptophan hydroxylase inhibitor, showed a significant and selective reduction of gut $5 \mathrm{HT}$ levels in a first trial on diarrheic IB [43].

New medications for visceral pain treatment include opioidagonists (e.g., asimadoline) effective on colonic distension pain, anticonvulsants (e.g., pregabalin) able to improve pain sensation through direct effect on visceral sensory pathways, glucagon-like peptide 1 inhibiting small intestinal migrating motor complexes and acting on nausea and pain, neurokinin antagonists (e.g., DNK333), NMDA antagonists (e.g., dextromethorphan) which might be helpful in reducing the "wind-up" phenomenon, antagonists of the transient receptor potential ion channel of the vanilloid type 1 as another promising viscero-analgesic agent [39,44-47]. Moreover, clinical studies have provided consistent evidence for an analgesic effect of the non-selective somatostatin receptors (widespread in CNS and gastrointestinal tract) agonist octreotide in clinical visceral pain models [39]. Last, anti-inflammatory drugs (e.g., ketotifen) have been recently tested with contrasting results in patients with visceral hypersensitivity [48].

Novel cannabinoid antagonists likely stimulating the leptin system have being recently investigated in the treatment of obesity and eating disorders, whereas the development of CCK antagonists might be helpful in the relief of early satiety in functional dyspepsia or inhibiting lower esophageal sphincter relaxation in patients with GERD $[49,50]$.

The consistent reports of hypervigilance in subjects with FGID have stimulated the research on adrenergic system modulators. The 2adrenergic receptor ( $\alpha 2 \mathrm{AR}$ ) agonists seem to exert their effect on anxiety, pain perception and autonomic system mainly through the action on the central noradrenergic pathways, whose role in IBS pathophysiology has been postulated. Clonidine has been suggested as a potential therapeutical tool in patients with lower functional bowel disorders, although side effects (e.g., fatigue) limit its usefulness [51]. The role of stress in FGID has been deeply studied and led to the search of medications acting on CRF1 and CRF2 receptors, whose action embraces neuroendocrine, autonomic, behavioural, and visceral responses to stress. In preliminary studies on animals and humans, the use of CRF receptor antagonists suggested a significant effect against stress-related behaviour, hyperalgesia, colonic secretion and motility, either via central and enteric pathways [52-54].

The discovery that antibiotic erythromycin acts as a motilinreceptor agonist, initiating phase III migrating motor complex activity and increasing gastrointestinal motility, promoted the development of motilin-receptor ligands in order to promote gastric emptying in gastroparesis and reducing food intake in severe obesity [55].

In conclusion, the ENS is a complex system with relative autonomy but high responsiveness to CNS and ANS modulation. The pathophysiology of BGA can be investigated with a growing number of tools effective on different levels of the axis, in agreement with the multifactorial features of its disorders. The knowledge of neurohormonal pathways represent a necessary step in the identification of effective and selective treatments for BGA dysfunctions.

\section{Role of the Funding Source}

No funding sources had any role in study design, collection, analysis and interpretation of data, or writing of the report. The 
corresponding author had full access to all the data of the study and had final responsibility for the decision to submit it for publication.

\section{Financial Disclosure}

None of the authors have any financial interest to disclose.

\section{References}

1. Benarroch EE (1993) The central autonomic network: functional organization, dysfunction, and perspective. Mayo Clin Proc 68: 988-1001.

2. Craig AD (2003) A new view of pain as a homeostatic emotion. Trends Neurosci 26: 303-307.

3. Critchley HD, Mathias CJ, Josephs O, O’Doherty J, Zanini S, et al. (2003) Human cingulate cortex and autonomic control: converging neuroimaging and clinical evidence. Brain 126: 2139-2152.

4. Mayer EA (2011) Gut feelings: the emerging biology of gut-brain communication. Nat Rev Neurosci 12: 453-466.

5. Aziz Q, Thompson DG (1998) Brain-gut axis in health and disease. Gastroenterology 114: 559-578.

6. Gillis RA, Quest JA, Pagani FD and Norman WP. Control centers in the central nervous system for regulating gastrointestinal motility. In: Schultz SG, Wood JD, Rauner BB, eds. Handbook of physiology. Section 6. The gastrointestinal system. New York: Oxford University, 1989: 621-683.

7. Cervero $\mathrm{F}$ and Foreman RD. Sensory innervation of the viscera. In Loewy AD, Spyer KM, eds. Central regulation of autonomic function. New York: Oxford University, 1990: 104-125.

8. Inskip JA, Ramer LM, Ramer MS, Krassioukov AV (2009) Autonomic assessment of animals with spinal cord injury: tools, techniques and translation. Spinal Cord 47: 2-35.

9. Goyal RK, Hirano I (1996) The enteric nervous system. N Engl J Med 334: 1106-1115

10. De Giorgio R, Sarnelli G, Corinaldesi R, Stanghellini V (2004) Advances in our understanding of the pathology of chronic intestinal pseudoobstruction. Gut 53: 1549-1552.

11. Martucciello G, Ceccherini I, Lerone M, Jasonni V (2000) Pathogenesis of Hirschsprung's disease. J Pediatr Surg 35: 1017-1025.

12. Drossman DA, Camilleri M, Mayer EA, Whitehead WE (2002) AGA technical review on irritable bowel syndrome. Gastroenterology 123: 2108-2131.

13. Bandlapalli P, Banji D, Banji OJF, Swetha D and Pratusha G (2011) Role of stress in pathophysiology of irritable bowel syndrome. IRJP 2: 54-60.

14. Bonaz BL, Bernstein CN (2013) Brain-gut interactions in inflammatory bowel disease. Gastroenterology 144: 36-49.

15. Thayer JF, Sternberg E (2006) Beyond heart rate variability: vagal regulation of allostatic systems. Ann N Y Acad Sci 1088: 361-372.

16. Bonaz B, Rivest S (1998) Effect of a chronic stress on CRF neuronal activity and expression of its type 1 receptor in the rat brain. Am J Physiol 275: R1438-1449.

17. Im E, Rhee SH, Park YS, Fiocchi C, Taché Y, et al. (2010) Corticotropinreleasing hormone family of peptides regulates intestinal angiogenesis. Gastroenterology 138: 2457-2467, 2467.

18. Coutinho SV, Plotsky PM, Sablad M, Miller JC, Zhou H, et al. (2002) Neonatal maternal separation alters stress-induced responses to viscerosomatic nociceptive stimuli in rat. Am J Physiol Gastrointest Liver Physiol 282: G307-G316.

19. Ghia JE, Blennerhassett $P$ and Collins SM (2008) Impaired parasympathetic function increases susceptibility to inflammatory bowel disease in a mouse model of depression. J Clin Invest 118: 2209-2218.

20. Heitkemper MM, Cain KC, Jarrett ME, Burr RL, Hertig V, et al. (2003) Symptoms across the menstrual cycle in women with irritable bowel syndrome. Am J Gastroenterol 98: 420-430.

21. Zhou Q, Verne GN (2011) New insights into visceral hypersensitivity-clinical implications in IBS. Nat Rev Gastroenterol Hepatol 8: 349-355.
22. Van Oudenhove L, Demyttenaere K Tack J and Aziz Q (2004) Central nervous system involvement in functional gastrointestinal disorders. Best Practice \& Research Clinical Gastroenterology 18: 663-680.

23. Van Oudenhove L, Coen SJ, Aziz Q (2007) Functional brain imaging of gastrointestinal sensation in health and disease. World J Gastroenterol 13: $3438-3445$

24. Rainville P (2002) Brain mechanisms of pain affect and pain modulation. Curr Opin Neurobiol 12: 195-204.

25. Sharma A, Lelic D, Brock C, Paine P, Aziz Q (2009) New technologies to investigate the brain-gut axis. World J Gastroenterol 15: 182-191.

26. Rössel P, Pedersen P, Niddam D, Arendt-Nielsen L, Chen AC, et al. (2001) Cerebral response to electric stimulation of the colon and abdominal skin in healthy subjects and patients with irritable bowel syndrome. Scand J Gastroenterol 36: 1259-1266.

27. Konturek SJ, Konturek JW, Pawlik T, Brzozowski T (2004) Brain-gut axis and its role in the control of food intake. J Physiol Pharmacol 55: 137-154.

28. Yüksel B (2009) Neuroendocrinological Control of Obesity. J Clin Res Ped Endo 1: 58-64.

29. Miller MJ, Sandoval M (1999) Nitric Oxide. III. A molecular prelude to intestinal inflammation. Am J Physiol 276: G795-799.

30. Barbara G, De Giorgio R, Stanghellini V, Cremon C, Corinaldesi R (2002) A role for inflammation in irritable bowel syndrome? Gut 51 Suppl 1: i41-44.

31. Camilleri M (2004) Is there a SERT-ain association with IBS? Gut 53: 1396-1399.

32. Hornby PJ (2001) Receptors and transmission in the brain-gut axis. II. Excitatory amino acid receptors in the brain-gut axis. Am J Physiol Gastrointest Liver Physiol 280: G1055-1060.

33. O'Hara AM, Shanahan F (2006) The gut flora as a forgotten organ. EMBO Rep 7: 688-693.

34. Collins SM, Surette M, Bercik P (2012) The interplay between the intestinal microbiota and the brain. Nat Rev Microbiol 10: 735-742.

35. Sparing R, Mottaghy FM (2008) Noninvasive brain stimulation with transcranial magnetic or direct current stimulation (TMS/tDCS)-From insights into human memory to therapy of its dysfunction. Methods 44 : 329-337.

36. Patrizi F, Freedman SD, Pascual-Leone A, Fregni F (2006) Novel therapeutic approaches to the treatment of chronic abdominal visceral pain. ScientificWorldJournal 6: 472-490.

37. O' Connell NE, Wand BM, Marston L, Spencer S and Desouza LH (2014) Non-invasive brain stimulation techniques for chronic pain. Cochrane Database Syst Rev 11: CD008208.

38. Vigneri S, Bonventre S, Inviati A, Schifano D, Cosentino G, et al. (2014) Effects of transcranial direct current stimulation on esophageal motility in patients with gastroesophageal reflux disease. Clin Neurophysiol

39. Mayer EA, Tillisch K, Bradesi S (2006) Review article: modulation of the brain-gut axis as a therapeutic approach in gastrointestinal disease. Aliment Pharmacol Ther 24: 919-933.

40. Camilleri M, Di Lorenzo C (2012) Brain-gut axis: from basic understanding to treatment of IBS and related disorders. J Pediatr Gastroenterol Nutr 54: 446-453.

41. Simrén M, Barbara G, Flint HJ, Spiegel BM, Spiller RC, et al. (2013) Intestinal microbiota in functional bowel disorders: a Rome foundation report. Gut 62: 159-176.

42. Guglielmetti S, Mora D, Gschwender M and Popp K (2011) Randomised clinical trial: Bifidobacterium bifidum MIMBb75 significantly alleviates irritable bowel syndrome and improves quality of life--a double-blind, placebo-controlled study. Aliment Pharmacol Ther 33: 1123-1132.

43. Camilleri M (2011) LX-1031, a tryptophan 5-hydroxylase inhibitor, and its potential in chronic diarrhea associated with increased serotonin. Neurogastroenterol Motil 23: 193-200.

44. Delgado-Aros S, Chial HJ, Camilleri M, Szarka LA, Weber FT, et al. (2003) Effects of a kappa-opioid agonist, asimadoline, on satiation and GI 
Citation: Vigneri S (2014) The Brain-Gut Axis: From Pathophysiology to Possible Future Strategies of Treatment. Brain Disord Ther 3: 137. doi: 10.4172/2168-975X.1000137

Page 7 of 7

motor and sensory functions in humans. Am J Physiol Gastrointest Liver Physiol 284: G558-566.

45. Iturrino J, Camilleri M, Busciglio I, Burton D, Zinsmeister AR (2011) Effect of the $\hat{\mathrm{I}} \pm 2 \hat{\mathrm{I}}^{\prime}$ ligand, pregabalin, on colonic sensory and motor functions in healthy adults. Am J Physiol Gastrointest Liver Physiol 301: G377-384.

46. Hellström PM, Hein J, Bytzer P, Björnssön E, Kristensen J, et al. (2009) Clinical trial: the glucagon-like peptide-1 analogue ROSE-010 for management of acute pain in patients with irritable bowel syndrome: a randomized, placebo-controlled, double-blind study. Aliment Pharmacol Ther 29:198-206.

47. Zhou Q, Price DD, Callam CS, Woodruff MA, Verne GN (2011) Effects of the N-methyl-D-aspartate receptor on temporal summation of second pain (wind-up) in irritable bowel syndrome. J Pain 12: 297-303.

48. Klooker TK, Braak B, Koopman KE, Welting O, Wouters MM, et al. (2010) The mast cell stabiliser ketotifen decreases visceral hypersensitivity and improves intestinal symptoms in patients with irritable bowel syndrome. Gut 59: 1213-1221.

49. Tonstad S (2006) Rimonabant: a cannabinoid receptor blocker for the treatment of metabolic and cardiovascular risk factors. Nutr Metab Cardiovasc Dis 16: 156-162.
50. Fried M, Feinle C (2002) The role of fat and cholecystokinin in functional dyspepsia. Gut 51 Suppl 1: i54-57.

51. Camilleri M, Kim DY, McKinzie S, Kim HJ, Thomforde GM, et al. (2003) A randomized, controlled exploratory study of clonidine in diarrheapredominant irritable bowel syndrome. Clin Gastroenterol Hepatol 1: 111-121.

52. Tache' Y, Martinez V, Million M and Maillot C (2002) Role of corticotropin releasing factor receptor subtype 1 in stress-related functional colonic alterations: implications in irritable bowel syndrome. Eur J Surg Suppl 587: 16-22.

53. Habib KE, Weld KP, Rice KC, Pushkas J, Champoux M, et al. (2000) Oral administration of a corticotropin-releasing hormone receptor antagonist significantly attenuates behavioral, neuroendocrine, and autonomic responses to stress in primates. Proc Natl Acad Sci USA 97: 6079-6084.

54. Sagami Y, Shimada Y, Tayama J, Nomura T, Satake M, et al. (2004) Effect of a corticotropin releasing hormone receptor antagonist on colonic sensory and motor function in patients with irritable bowel syndrome. Gut 53: 958-964.

55. Sanger GJ, Lee K (2008) Hormones of the gut-brain axis as targets for the treatment of upper gastrointestinal disorders. Nat Rev Drug Discov 7: 241-254. 